\title{
BMJ Open New model of integrated care for uncontrolled type 2 diabetes in a retrospective, underserved adult population in the USA: a study protocol for an effectiveness and cost- effectiveness analysis
}

Rita Bosetti (D) , ${ }^{1}$ Laila Tabatabai, ${ }^{2}$ George Naufal, ${ }^{1,3}$ Rosbel Brito, ${ }^{4}$ Bita Kash ${ }^{1,5}$

To cite: Bosetti R, Tabatabai L, Naufal G, et al. New model of integrated care for uncontrolled type 2 diabetes in a retrospective, underserved adult population in the USA: a study protocol for an effectiveness and cost-effectiveness analysis. BMJ Open 2020;10:e038084. doi:10.1136/ bmjopen-2020-038084

- Prepublication history for this paper is available online To view these files, please visit the journal online (http://dx.doi. org/10.1136/bmjopen-2020038084).

Received 26 February 2020 Revised 29 June 2020 Accepted 01 July 2020
Check for updates

(c) Author(s) (or their employer(s)) 2020. Re-use permitted under CC BY-NC. No commercial re-use. See rights and permissions. Published by BMJ.

For numbered affiliations see end of article.

Correspondence to

Dr Rita Bosetti;

rbosetti@houstonmethodist.org

\section{ABSTRACT}

Introduction Type 2 diabetes prevalence is increasing in the USA, especially in underserved populations. Patient outcomes can be improved by providing access to specialty care within Federally Qualified Health Centers, possibly improving the cost-effectiveness of diabetes care.

Methods and analysis A new model of diabetes care based on multidisciplinary teams of clinical fellows, supported by an endocrinologist for underserved adult populations, is presented. The study uses a retrospective, non-randomised cohort of patients with diabetes who visited the community clinic between 1 January 2012 and 31 December 2018. A quasi-experimental method to analyse the causal evidence of the effect of the new model is presented. Discontinuity regression is used to compare two interventions, the intervention by a Clinical Fellow Endocrinology Programme and usual care by a primary care physician. Patients are referred to the Clinical Fellow Endocrinology Programme in case of uncontrolled diabetes (glycated haemoglobin $(\mathrm{HbA} 1 \mathrm{c}) \geq 9 \%)$. The regression discontinuity design allows the construction of a treatment group for patients with an $\mathrm{HbA1c}$ equal or above the threshold in comparison with a control group for patients with an $\mathrm{HbA1c}$ below the threshold. The patient outcomes and cost-effectiveness of the new model are analysed. Regression models will be used to assess the differences between treatment and control groups.

Ethics and dissemination Quantitative patient data are received by the study team in a de-identified format for analysis via an institutional review board-approved protocol. The quantitative study has been approved by the Houston Methodist Research Institute Institutional Review Board, Houston, Texas, USA. Anticipated results will not only provide evidence about the impact of patient outcomes in underserved diabetic populations, but also give an idea of the cost-effectiveness of the new model and whether or not cost savings can be attained for patients, third-party payers and society. The results will help set up evidence-based policy guidelines in diabetes care. Results will be disseminated through papers, conferences and public health/policy fora.
Strengths and limitations of this study

- This analysis addresses the short-term and longterm cost-effectiveness of a new model of diabetes care, which is critical to inform the care pathway of underprivileged diabetes patients.

- The cost analysis accounts for multiple stakeholders (patients, third-party payers and society) and a wide range of direct and indirect sources of cost and benefit.

- Cost analyses of innovative programmes make it possible to establish more general evidence-based policy guidelines for broader implementation and impact.

- Allocation in the original referral programme was non-randomised, and thus, retrospective comparison between patient groups cannot wholly exclude selection bias.

\section{INTRODUCTION}

Type 2 diabetes mellitus (T2DM), a systemic disease characterised by hyperglycaemic caused by impaired glucose tolerance, impaired fasting glucose or both, ${ }^{1}$ is the most common chronic disease affecting more than 30 million adults in the USA. ${ }^{2}$ Although increasing age, shifting ethnic demographics and a rising number of obese individuals cause an increase in the incidence of diabetes, ${ }^{3}$ the highest prevalence of the disease can be found among the uninsured. ${ }^{4-7}$ The management of T2DM is burdensome and may cause one or more long-term microvascular and macrovascular complications, such as cardiovascular disease,${ }^{8-15}$ ischaemic stroke ${ }^{8913}$ eye disorders and blindness, ${ }^{891415}$ foot ulcers and amputations, ${ }^{891415}$ neuropathy ${ }^{81415}$ and chronic kidney disease. ${ }^{891415}$ Besides emotionally and physically overwhelming patients, 
diabetes has large impacts on society. This is reflected in the US burden for diabetes, which was estimated at US $\$ 327$ billion in $2017 .{ }^{16}$ Costs increased by more than $25 \%$ over a period of 5 years-since the previous estimate was set at US $\$ 245$ billion in $2012 .{ }^{16}$ Costs as a consequence of diabetes also include increased absenteeism (US $\$ 3.3$ billion), reduced productivity at work (US\$26.9 billion) for the population at work, reduced productivity for those not in the labour workforce (US\$2.3 billion), inability to work as a result of diseaserelated disability (US $\$ 37.5$ billion) and lost productive capacity from premature mortality (US $\$ 19.9$ billion). These are the indirect diabetes-related costs and are included in the US burden for diabetes. ${ }^{16}$ For individual patients, diabetes has a significant financial impact. In general, patients with diabetes incur average medical expenditures of US $\$ 16752$ per year-US $\$ 9601$ attributed to diabetes. ${ }^{16}$ On average, medical expenditures of people with diabetes are approximately 2.3 times higher than for those without diabetes. ${ }^{16}$ The largest components of the medical bill of diabetes patients are the following: hospital inpatient care $(30 \%)$, prescription medications to treat hyperglycaemic and its long-term complications $(30 \%)$, antidiabetic agents and diabetes supplies $(15 \%)$, and physician office visits (13\%). ${ }^{16}$

In the USA, medically underserved individuals-persons with poor or no health insurance-have limited access to specialty care and prescription drugs, increasing the risk of diabetes and long-term complications in these populations. ${ }^{17}$ According to the Centers for Disease Control and Prevention and the National Center for Health Statistics, the number of Medicaid-eligible individuals sharply increased in 2013-2014. ${ }^{18}$ In 2016, however, there was a new drop-in person coverage by government health insurance, particularly among the Hispanic community. ${ }^{18}$

With appropriate support from community health centres, medically underserved individuals can receive care, nutrition counselling, low-cost medications and patient support. ${ }^{19}$ In the USA, different diabetes selfmanagement programmes have been set up for underserved populations, demonstrating improvements in glycated haemoglobin (HbAlc), cholesterol, blood pressure, body mass index (BMI) and health-related quality of life. ${ }^{20-34}$ Programmes involving diabetes management through community health workers, telephone-based diabetes management, diabetes self-management, teambased care management and quality improvement collaboratives were offered and studied in underserved US populations. ${ }^{20-34}$ However, no programme in the USA has assessed diabetes interventions conducted by specialty care in underserved populations, which could take on a much greater role in diabetes management to help meet the current demands and needs in medically underserved populations. Moreover, the real cost of diabetes management interventions for underserved populations in the USA has never fully been assessed.

The specific objective of this study is to assess the patient outcomes and the cost-effectiveness of a new integrated model of care for medically underserved populations. Although different patient outcomes are assessed, the primary outcome is HbA1c. Secondary outcomes include: blood pressure, cholesterol, BMI as well as the costeffectiveness of the intervention.

\section{METHODS AND ANALYSIS \\ Study design}

We report our study design according to the methods section of the Strengthening the Reporting of Observational Studies in Epidemiology statement for cohort studies. ${ }^{35}$ The retrospective study is based on patient records from a non-randomised cohort linked to the diagnosis of T2DM with either referral or non-referral to a specialty programme, the Clinical Fellow Endocrinology Programme, which started in 2014. To define the treatment and control groups, a regression discontinuity approach is used. Patients with an HbAlc value of at least the threshold of $9 \%$ (or $75 \mathrm{mmol} / \mathrm{mol}$ ) have been systematically recommended to the Clinical Fellow Endocrinology Programme. Consequently, the regression discontinuity approach makes it possible to create a treatment group (patients with an HbA1c equal and above threshold) and compare it with a control group (patients with an HbA1c below threshold). Such panel data allow us to control for unobservable factors that do not change over time, but change per patient (patient-specific factors).

The research questions include:

1. Does an Endocrinology Programme for patients at a community clinic improve health outcomes in patients with diabetes compared with usual care?

2. Does an Endocrinology Programme for patients at a community clinic improve economic outcomes in patients with diabetes compared with usual care? Does it improve economic outcomes for society?

3. What is the reciprocal cost-effectiveness ratio of the Endocrinology Programme compared with usual care? How much change (improvement) in health outcomes is achieved per US $\$ 1000$ spent?

\section{Study setting}

The study is conducted within a Federally Qualified Health Center setting. Both the intervention (Clinical Fellow Endocrinology Programme) and usual care (primary care physician (PCP)) were administered to patients at a community clinic in the Montrose district, Houston, Texas, USA. The community clinic in Montrose is a 40000 square foot, state-of-the-art clinic, serving primarily underserved individuals. The clinic offers highquality care for individuals with a low income, addressing their unmet medical needs. Patients at the clinic are $80 \%$ Hispanic, 10\% African American and 10\% Caucasian and Asian. More than $97 \%$ of patients are poorly insured or non-insured.

\section{Participants}

Patients with uncontrolled T2DM (HbA1c $\geq 9 \%)$ were eligible to be referred to the Clinical Fellow Endocrinology 
Programme. Patients with an $\mathrm{HbAlc}<9 \%$ were treated by the PCP at the community clinic. Once referred to the clinical fellow because initial HbAlc is uncontrolled, patients remain under treatment of the clinical fellow even in case their HbAlc improves and diabetes becomes controlled. Only uninsured individuals could be referred to the Clinical Fellow Endocrinology Programme. The participants of this analysis are identified retrospectively from the community clinic. Patients with diabetes who visited the community clinic from 2012 to 2018 are considered in the study. Before the Clinical Fellow Endocrinology Programme started in 2014, patients with uncontrolled diabetes were seen by PCPs. De-identified data of these patients will be provided by the data management team of the community clinic. After receiving the data, analyses will start.

\section{Inclusion and exclusion criteria}

The Clinical Fellow Endocrinology Programme started in 2014, but in our study, we include all adult patients $(\geq 18$ years of age), who visited the community clinic between 1 January 2012 and 31 December 2018, diagnosed with diabetes (HbAlc $>6 \%$ or $42 \mathrm{mmol} / \mathrm{mol}$ ) or uncontrolled diabetes (HbA1c $\geq 9 \%)$ and the requirement of no health insurance. This allows us to assess the effectiveness of pre and post programme. Exclusion criteria included patients younger than 18 years of age, patients who had some form of insurance (eg, Medicaid, Medicare or private insurance), patients diagnosed with type 1 or gestational diabetes. Also pregnant patients are excluded from the analyses.

All included patients were treated by a clinical endocrinology fellow or a PCP, depending on the severity of diabetes. Patients with uncontrolled diabetes (HbAlc $\geq 9 \%$ ) were referred to the Clinical Fellow Endocrinology Programme at the community clinic. Patients with controlled diabetes $(\mathrm{HbA} 1 \mathrm{c}<9 \%)$ were treated by a PCP.

\section{Contexts of the referral process and Clinical Fellow Endocrinology} Programme

Staff located at the community clinic determined patients' eligibility through patient forms. The patients' basic demographic data were also collected at the first appointment at the community clinic. Simple walk-ins were not permitted. After receiving confirmation of their eligibility, patients were assigned a Medical Record Number. Eligible patients were seen by a nurse practitioner or physician for the first basic screening and referred to a PCP at the community clinic for a next medical visit. All eligible patients were seen by a PCP, whereas those diagnosed with uncontrolled diabetes (HbAlc $\geq 9 \%$ ) were systematically referred to a fellow of the Clinical Fellow Endocrinology Programme for further care. After referral, the patients meet with the nurse, who collected some additional health indicators from them and scheduled an initial appointment with the clinical fellow. On that appointment, follow-up appointments are also scheduled. Based on the severity of the patients' diabetes, follow-up was scheduled at least every 3 months.

\section{Intervention}

Patients in the intervention arm were treated by a multidisciplinary endocrinology team at the community clinic and consisted of nurses, PCPs, clinical endocrinology fellows and a supervising endocrinologist. Clinical fellows are MDs who have completed an internal medicine residency and are pursuing further postgraduate training in endocrinology. They are doing their training at a teaching hospital and work at the community clinic once a week under the supervision of the endocrinologist. The patient, once referred, receives counselling and support, as well as a review of medications, patient's history of diabetes, depression screening, blood and urine testing, and nutritional counselling. The clinical fellow helps the patient address issues associated with diabetes, such as glycaemic control, blood pressure, cholesterol, the patient's lifestyle and the management of diabetes complications. The clinical fellow discusses everything with the supervising endocrinologist before finalising with the patient. Depending on the severity of diabetes, follow-up is advised at least every 3 months.

\section{Usual care}

Usual care is provided at the community clinic to patients without insurance and without uncontrolled diabetes $(\mathrm{HbAlc}<9 \%)$. Patients in the usual care group were treated by their PCP, after a first medical screening visit. Also, patients in the usual care arm were given counselling and support by the team of the community clinic. Furthermore, the team reviewed the patients' medications, patient's history of diabetes, depression screening, blood and urine testing, and nutritional counselling. Follow-up is advised for these patients once every 3 months.

\section{Quantitative study data}

The collection of quantitative data will include: (1) patient demographics received from the data management team of the community clinic; and (2) clinical data also received directly from the community clinic. Both demographic and clinical data will be received in a de-identified format. These data are received for all diabetes patients who visited the community clinic in the 7-year period under study.

\section{Patient demographics}

De-identified patient demographics include the following: patient ID, age, gender at birth, current gender race, ethnicity, education level, income level, marital status, employment status and insurance status.

\section{Clinical indicators}

The primary clinical outcome measure is glycaemic control, measured by the mean change in HbAlc. Secondary clinical endpoints included in the study are: BMI, weight, blood pressure, heart rate, total cholesterol, 
low-density lipoprotein, high-density lipoprotein, total triglycerides, urine microalbumin, glucose levels, blood urea nitrogen (BUN), creatinine levels and glomerular filtration rate. Clinical data are received directly from the data management team of the community clinic. Information on patients' diabetes complications will be received as well. If applicable, we will also receive information on medications, health services use and date of each visit.

\section{Economic indicators}

In this study, we will use a comprehensive cost model, an innovative way of calculating costs in healthcare. Including all costs is crucial to avoid suboptimal policy recommendations. As indirect costs can have major impacts on total costs, direct and indirect costs of both interventions are considered, regardless of who bears them. This methodology is also recommended by the Second Panel on CostEffectiveness in Health and Medicine. ${ }^{36}$ Direct and indirect costs for each patient will be calculated from the societal perspective. The direct costs of diabetes care include: (1) drug costs (cost of study drug, costs to treat long-term microvascular and macrovascular complications, costs of drugs to treat therapy-related adverse events and costs for materials to administer drugs); (2) administration costs (inpatient costs and outpatient costs); (3) monitoring costs (costs for diagnosis and cost for follow-up); (4) costs for medical care (costs for medical visits, costs for emergency room visits and costs for specialty care visits); (5) expected costs for drug administration at home by a nurse; (6) costs for additional therapies; and (7) the expected costs for aftercare (rehabilitation, palliative care and psychological assistance). Costs are expected costs when they are based on an estimated number of patients using a specific medical service. Direct medical costs data will be collected and acquired in collaboration with the community clinic. Other direct costs will be collected from a Texas State database-The Texas Inpatient Public Use Data File. ${ }^{37}$ This database contains data on discharges from Texas hospitals. In addition, the following indirect costs of diabetes care will be included: (1) productivity losses (including lost income for patients and expected lost income of unpaid caretakers); (2) expected costs for paid caretakers; and (3) transportation costs (including travel costs and parking expenses). Work productivity losses will be estimated by estimating lost workdays and using US census level estimates for wages (along with employment identification from the data from the community clinic) to estimate lost earnings. The economic analysis will comprise a cost-effectiveness analysis. Table 1 gives an overview of the comprehensive cost model for diabetes care interventions. Costs will be measured over the period of the study as well as over the long-term. To estimate the lifetime cost-effectiveness, simulation modelling will be used to project lifetime incremental health effects and costs based on changes in HbAlc, using the Archimedes model. This clinically detailed and validated model simulates the effects of interventions by generating lifetime forecasts in health outcomes and costs. The model encompasses human physiology, disease progression and healthcare delivery. Comparisons are made with a hypothetical group of patients with similar demographics as the sample population. We will evaluate the independent effect of the following diabetes complications: cardiovascular disease, stroke, eye disease, blindness, hypoglycaemic, nephropathy, neuropathy, foot ulcer, amputation, ketoacidosis and mortality. To estimate the lifetime cost-effectiveness of the Clinical Fellow Endocrinology Programme, we will create a simulated population based on real population data from the National Health and Nutrition Survey, ${ }^{38}$ which is representative for the general US population. The number of subjects included in each arm of the simulation will depend on the sensitivity of the analysis to the number of simulated individuals. Both costs and health outcomes will be discounted at $3 \%$ annually. Only costs that differ between the treatment and control arm will be included in the analysis.

\section{Sample size}

The number of adult patients seen at the community clinic is around 25000 per year. Over the study period 2012-2018, the clinic saw 175000 patients for all conditions. Assuming the fact that individuals of ethnic minorities and low socioeconomic status are disproportionately affected by diabetes-such as the patients of the community clinic-we assume that around $14 \%$ of patients have a diagnosis of diabetes. ${ }^{39} 40$ Therefore, our sample size will be estimated at $(175000 * 0.14)$ or 24500 patients in the period 2012-2018. Of these patients, we expect that approximately $10 \%$ will have at least one follow-up visit, thus around 2450 patients are included in the study. For this sample of 2450 patients, from the community clinic's internal database, we can assume that approximately $50 \%$ of patients will be excluded because they will have some form of insurance (Medicaid, Medicare, other public insurance or private insurance). Furthermore, a small percentage (approximately 10\%) of patients will be excluded because they are pregnant at the moment of diagnosis. We estimate our final sample size will be around 1100 patients.

\section{Statistical analysis plan}

The selection into the Clinical Fellow Endocrinology Programme is not random, but determined by the PCP who will, on assessment (if $\mathrm{HbAlc} \geq 9 \%$ ), decide whether to recommend the patient to the clinical fellow or not. We exploit a sharp discontinuity around the levels of HbA1c at the community clinic. The community clinic PCP diverts patients with diabetes to the Clinical Fellow Endocrinology Programme if their HbAlc level is at least 9\%. This discontinuity allows us to identify a control group to key out the causal effect of the Clinical Fellow Endocrinology Programme. The control group will be formed of patients with diabetes with levels of HbAlc below the $9 \%$ threshold, with an implicit assumption that the threshold is arbitrary and hence as good as random. The 
Table 1 Comprehensive cost model for diabetes care interventions

\section{Direct costs}

Diagnosis and treatment

1. Drug costs

Study drug: diabetes treatment

Drugs to treat long-term macrovascular and microvascular complications

Drugs to treat therapy-related adverse events

Materials to administer drugs

\begin{tabular}{|c|c|}
\hline \multirow[t]{2}{*}{ 2. Administration costs } & Inpatient costs \\
\hline & Outpatient costs (day hospitalisations) \\
\hline \multirow[t]{2}{*}{ 3. Monitoring costs } & Diagnosis \\
\hline & Materials and equipment for diagnostic and follow-up tests \\
\hline 4. Additional visits & Medical visits \\
\hline 5. Expected home care costs & Expected drug administration at home by a nurse \\
\hline \multirow[t]{5}{*}{ 6. Additional therapies } & Myocardial infarction \\
\hline & Stroke \\
\hline & Heart failure and heart disease \\
\hline & Ketoacidosis \\
\hline & Pancreatitis \\
\hline \multirow[t]{3}{*}{ 7. Expected after care costs } & Psychological assistance \\
\hline & Rehabilitation \\
\hline & Palliative care \\
\hline
\end{tabular}

\section{Indirect costs}

\section{Productivity losses}

\section{Expected costs for paid caregivers}

\section{Transportation costs}

Income loss for the patient

Expected income loss for unpaid caregivers

Paid income to external caregivers (based on average hourly wage)

Travel expenses per kilometre/mile

Parking expenses identifying presumption is that all factors explaining the outcomes of patients with diabetes should vary smoothly around the HbAlc threshold and patient or staff manipulation cannot occur. Such a setting is ideal for a regression discontinuity design. We test for this identification strategy by examining whether observable patient characteristics do not vary discontinuously across the HbAlc threshold. We plan to use the same approach to identify the effects on different patient populations to examine whether the Clinical Fellow Endocrinology Programme's impact is heterogeneous across different populations. We identify gender, age groups and race.

The cut-off $\mathrm{HbA1c}$ will be at $9 \%$. The predictor variable in our research is a dummy 1 if a patient has seen a fellow from the Clinical Fellow Endocrinology Programme and 0 otherwise. Control variables include demographics of the patient, comorbidities and fellow characteristics (age, gender at birth, race, ethnicity, education level, income level, marital status, employment status, family size, homelessness status, insurance status, smoking status, alcohol use, primary language, depression, hypertension, retinopathy, neuropathy, nephropathy, cardiovascular disease, cerebrovascular disease, peripheral vascular disease, hyperlipidaemia and end-stage renal disease). Furthermore, the variables HIV, lesbian-gay-bisexualtransgender and history of sex reassignment surgery are identified as possible confounders in the data set. These unobservable variables could distort the association between the independent and dependent variables. To control for possible confounding effects in the sample population, we will perform subpopulation analyses. We are not sure about how many patients will have an HbAlc 
of $9 \%$, counting those at $9 \%$ to be part of the treatment group. Part of the sensitivity analysis and robustness checks, we plan to exclude these from the treatment and running the same analysis. We will use the mean square error optimal bandwidth as the functional form and we will also check for sensitivity around bandwidths choice.

We will work with the community clinic to identify whether missing data come from non-random patterns or whether they are completely random (due to, eg, data system issues). If the reasons are random, then we will proceed with only available data in the analysis. If the missing pattern is non-random, then we will either identify groups in which data are more complete or investigate data imputation techniques. We will identify outliers and run both outlier-included and outlier-excluded analyses.

The demographic and clinical characteristics of participants at baseline will be compared between intervention and control groups. We will analyse the primary clinical outcome variable, HbAlc, by using linear regression. Linear or logistic regression will be used to assess the continuous and binary secondary outcome variables, respectively. Analyses will be performed on an intentionto-treat basis. Statistical significance is reached when $\mathrm{p}<0.05$. As we will report according to the Consolidated Health Economic Evaluation Reporting Standards guidelines ${ }^{41}$ it is important to note that the data we will receive from the community clinic comprise patients who were diagnosed with T2DM from 2012 to 2018. The data include multiple observations per patient; each observation represents a visit to the community clinic. Data cleaning and management will be performed to create a longitudinal data set of patients with visits to the community clinic.

\section{Patient and public involvement}

As the quantitative aims rely on de-identified retrospective data and no direct contact with patients (depending in part on the undisturbed flow of clinic operations to form the natural comparison groups), patients and public are not involved directly in this study. As a research team, we will receive a de-identified data set directly from the data management team of the community clinic. After study termination, study results will be shared with the community clinic, who will communicate the results with the patients.

\section{DISCUSSION}

A protocol for the study design and methods to evaluate a new model of uncontrolled T2DM care in underserved US adult populations is presented. The protocol examines a collaboration between a community clinic and a highly specialised Clinical Fellow Endocrinology Programme through a teaching hospital. The programme is hypothesised to facilitate the access of specialty care to underserved populations, improving the quality and safety of healthcare for these patients with uncontrolled T2DM. This is the first study in the USA comparing the usual care (treatment by a PCP) with an intervention giving underserved patients with uncontrolled diabetes (HbAlc $\geq 9 \%$ ) access to specialty care. Other advantages, such as cost savings, on the longer term could also be attained by avoiding costly diabetes complications in underserved populations.

While the fundamental goal of healthcare is to improve health in general, the results of this study could lead to new guidelines leading to improved health for medically underserved populations, who have otherwise limited access to specialty care. This study will not only provide empirical evidence about the impact of health outcomes on medically underserved diabetic populations in the USA, but will also give a broader idea of the short-term and long-term cost-effectiveness of the new model of care and whether cost savings can be attained. The results will help to establish evidence-based policy guidelines in diabetes care. If cost-effective, then the new model of care could improve patient outcomes in many underserved patients nationwide while having positive effects on society.

The study has some limitations that are important to mention. As the study is a non-randomised study, selection bias cannot be excluded. As only patients with uncontrolled T2DM (HbA1c $\geq 9 \%)$ are referred to the Clinical Fellow Endocrinology Programme, a discontinuity regression design was used to limit selection bias. Although we will control for confounding by using the discontinuity regression design, patients may have been allowed into the Clinical Fellow Endocrinology Programme without having the necessary requirements. Therefore, confounding by selection bias cannot be excluded due to unobservable factors. There may also be some patient issues around the follow-up. Medically underserved individuals tend not to return for follow-up visits and so dropout may be high. Drop outs after recruitments are a problem as they affect the generalisability of the conclusions of the study, affecting the internal validity. Literature suggests that attrition rates within 20\% are acceptable in diabetes management interventions ${ }^{42}$; our recently conducted literature search suggests that in selfmanagement diabetes interventions for underserved US populations, almost all studies have attrition rates equal to or smaller than $20 \%$. Therefore, we expect that our data set will also have this characteristic. Furthermore, it would be important to account for differential loss-tofollow-up as this could influence the study results. As this is a retrospective study, we cannot control for differential loss-to-follow-up. However, we can minimise its effects through propensity score matching. The propensity score will weight and match the population on control and intervention groups. This means that patients in the PCP setting will be given larger weights compared with the clinical fellow settings (given less weight in the matched data). Consequently, we will run our models on matched data. In our study, we will match on a 1:2 ratio, meaning one intervention patient for every two control patients. We will run generalised linear or logistic regression on matched data. Additionally, cost data may be difficult to 
obtain, as usually claims or prices paid by patients are registered. Nevertheless, every effort to obtain the cost of drugs and services will be made. If necessary, we will combine the information of national databases to the information of our study.

Finally, a future direction for the study is to collect patient-reported and employee-reported outcomes collected through the use of questionnaires to supplement the retrospectively available data. Data will be collected at baseline and at a 12-month follow-up, pending additional institutional review board (IRB) approval. Patients and fellows will be randomly selected to take part in the qualitative data collection portion. The objective will be to attain an overview of their experiences with the Clinical Fellow Endocrinology Programme. As for patients we want to know if the quality of life improved after having access to the programme, the Short Form 36 (SF-36) will be distributed to 200 patients in the intervention group (balanced for age, sex and HbA1c) through the community clinic's nurse. With the SF-6D, we will be able to uniquely classify each patient who completes the SF-36. Written consent forms will be distributed to participants prior to the data collection. Consent forms will highlight the voluntary nature and anonymity of the participation.

\section{Ethics and dissemination}

The quantitative study has been approved by the Houston Methodist Research Institute Institutional Review Board (Pro00020540), Houston, Texas, USA. We will receive the demographic and clinical patient data directly from the data management team of the community clinic in a de-identified format, and so consent for analysis of de-identified data was determined unnecessary.

Next to the publication of results in peer-reviewed scientific journals, expected to occur after the research has been completed, we plan to present the results of this work also at international conferences. After publication or presentation at conferences, the study findings will be freely shared on health policy fora with the objective to improve healthcare quality in medically underserved populations in the USA.

\author{
Author affiliations \\ ${ }^{1}$ Center for Outcomes Research, Houston Methodist Research Institute, Houston, \\ Texas, USA \\ ${ }^{2}$ Division of Endocrinology, Houston Methodist Hospital, Houston, Texas, USA \\ ${ }^{3}$ Public Policy Research Institute, Texas A\&M University System, College Station, \\ Texas, USA \\ ${ }^{4}$ Office of Graduate Medical Education, Houston Methodist Research Institute, \\ Houston, Texas, USA \\ ${ }^{5}$ School of Public Health, Texas A\&M University System, College Station, Texas, USA
}

Acknowledgements The authors want to thank JM Kolman for his writing assistance. No patients or patient advisers were directly involved in the study.

Contributors RB and GN conceived the study protocol and were responsible for the research design and statistical analysis plan. RB prepared the initial draft of the manuscript and then GN, LT, RBr and BK did critical revision. RB drafted the final draft of the paper, while $\mathrm{LT}, \mathrm{GN}, \mathrm{RBr}$ and $\mathrm{BK}$ helped to critically revise successive drafts of the manuscript.
Funding This work was partially supported through a grant from 0ccidental Petroleum.

Competing interests None declared.

Patient and public involvement Patients and/or the public were not involved in the design, or conduct, or reporting, or dissemination plans of this research.

Patient consent for publication Not required.

Provenance and peer review Not commissioned; externally peer reviewed.

Open access This is an open access article distributed in accordance with the Creative Commons Attribution Non Commercial (CC BY-NC 4.0) license, which permits others to distribute, remix, adapt, build upon this work non-commercially, and license their derivative works on different terms, provided the original work is properly cited, appropriate credit is given, any changes made indicated, and the use is non-commercial. See: http://creativecommons.org/licenses/by-nc/4.0/.

ORCID iD

Rita Bosetti http://orcid.org/0000-0002-0398-2834

\section{REFERENCES}

1 American Diabetes Association. Diagnosis and classification of diabetes mellitus. Diabetes Care 2014;37 Suppl 1:S81-90.

2 American Diabetes Association. Economic costs of diabetes in the U.S. in 2012. Diabetes Care 2013;36:1033-46.

3 Vijan S. Type 2 diabetes. Ann Intern Med 2010;152:ITC3-1-1.

4 Mehta PP, Santiago-Torres JE, Wisely CE, et al. Primary care continuity improves diabetic health outcomes: from free clinics to federally qualified health centers. J Am Board Fam Med 2016;29:318-24.

5 Agardh EE, Sidorchuk A, Hallqvist J, et al. Burden of type 2 diabetes attributed to lower educational levels in Sweden. Popul Health Metr 2011;9:60.

6 Berkowitz SA, Meigs JB, DeWalt D, et al. Material need insecurities, control of diabetes mellitus, and use of health care resources: results of the measuring economic insecurity in diabetes study. JAMA Intern Med 2015;175:257-65.

7 Booth GL, Hux JE. Relationship between avoidable hospitalizations for diabetes mellitus and income level. Arch Intern Med 2003;163:101-6.

8 Crandall JP, Knowler WC, Kahn SE, et al. The prevention of type 2 diabetes. Nat Clin Pract Endocrinol Metab 2008;4:382-93.

9 Diabetes Control and Complications Trial Research Group, Nathan DM, Genuth S, et al. The effect of intensive treatment of diabetes on the development and progression of long-term complications in insulin-dependent diabetes mellitus. N Engl J Med 1993;329:977-86.

10 The ACCORD Study Group. Effects of intensive blood-pressure control in type 2 diabetes mellitus. N Engl J Med Overseas Ed 2010;362:1575-85.

11 Heart Outcomes Prevention Evaluation (HOPE) Study Investigators. Effects of ramipril on cardiovascular and microvascular outcomes in people with diabetes mellitus: results of the HOPE study and MICRO-HOPE substudy. heart outcomes prevention evaluation study Investigators. Lancet 2000;355:253-9.

12 Scirica BM, Bhatt DL, Braunwald E, et al. Saxagliptin and cardiovascular outcomes in patients with type 2 diabetes mellitus. $N$ Engl J Med 2013;369:1317-26.

13 Emerging Risk Factors Collaboration, Sarwar N, Gao P, et al. Diabetes mellitus, fasting blood glucose concentration, and risk of vascular disease: a collaborative meta-analysis of 102 prospective studies. Lancet 2010;375:2215-22.

14 Boulé NG, Haddad E, Kenny GP, et al. Effects of exercise on glycemic control and body mass in type 2 diabetes mellitus: a metaanalysis of controlled clinical trials. JAMA 2001;286:1218-27.

15 Yki-Järvinen $\mathrm{H}$. Toxicity of hyperglycaemia in type 2 diabetes. Diabetes Metab Rev 1998;14 Suppl 1:S45-50.

16 American Diabetes Association. Economic costs of diabetes in the U.S. in 2017. Diabetes Care 2018:dci180007.

17 Philis-Tsimikas A, Walker C. Improved care for diabetes in underserved populations. J Ambul Care Manage 2001;24:39-43.

18 Cohen RA. Long-term trends in health insurance: Estimates from the National Health Interview Survey, United States, 1968-2017. U.S. Department of Health and Human Services, Centers for Disease Control and Prevention, 2018. Available: https://www. cdc.gov/nchs/data/nhis/health_insurance/TrendHealthlnsur ance1968_2017.pdf [Accessed 24 Sep 2019].

19 Shaw BA, Gallant MP, Riley-Jacome M, et al. Assessing sources of support for diabetes self-care in urban and rural underserved communities. J Community Health 2006;31:393-412. 
20 Brown SA, Blozis SA, Kouzekanani K, et al. Dosage effects of diabetes self-management education for Mexican Americans: the Starr County border health Initiative. Diabetes Care 2005;28:527-32.

21 Scanlon DP, Hollenbeak CS, Beich J, et al. Financial and clinical impact of team-based treatment for Medicaid enrollees with diabetes in a federally qualified health center. Diabetes Care 2008;31:2160-5.

22 Schechter CB, Cohen HW, Shmukler C, et al. Intervention costs and cost-effectiveness of a successful telephonic intervention to promote diabetes control. Diabetes Care 2012;35:2156-60.

23 Huang SJ, Galárraga O, Smith KA, et al. Cost-Effectiveness analysis of a cluster-randomized, culturally tailored, community health worker home-visiting diabetes intervention versus standard care in American Samoa. Hum Resour Health 2019;17:17.

24 Smith KJ, Hsu HE, Roberts MS, et al. Cost-Effectiveness analysis of efforts to reduce risk of type 2 diabetes and cardiovascular disease in southwestern Pennsylvania, 2005-2007. Prev Chronic Dis 2010;7:A109.

25 Brownson CA, Hoerger TJ, Fisher EB, et al. Cost-Effectiveness of diabetes self-management programs in community primary care settings. Diabetes Educ 2009;35:761-9.

26 Gilmer T, O'Connor PJ, Schiff JS, et al. Cost-Effectiveness of a community-based diabetes prevention program with participation incentives for Medicaid beneficiaries. Health Serv Res 2018;53:4704-24.

27 McAdam-Marx C, Dahal A, Jennings B, et al. The effect of a diabetes collaborative care management program on clinical and economic outcomes in patients with type 2 diabetes. J Manag Care Spec Pharm 2015;21:452-68.

28 Prezio EA, Pagán JA, Shuval K, et al. The community diabetes education (code) program: cost-effectiveness and health outcomes. Am J Prev Med 2014:47:771-9.

29 Krukowski RA, Pope RA, Love S, et al. Examination of costs for a lay health educator-delivered translation of the diabetes prevention program in senior centers. Prev Med 2013;57:400-2.

30 Ryabov I. Cost-Effectiveness of community health workers in controlling diabetes epidemic on the U.S.-Mexico border. Public Health 2014;128:636-42.
31 Brown HS, Wilson KJ, Pagán JA, et al. Cost-Effectiveness analysis of a community health worker intervention for low-income Hispanic adults with diabetes. Prev Chronic Dis 2012;9:E140.

32 Huang ES, Zhang Q, Brown SES, et al. The cost-effectiveness of improving diabetes care in U.S. federally qualified community health centers. Health Serv Res 2007;42:2174-93.

33 Gilmer TP, Roze S, Valentine WJ, et al. Cost-Effectiveness of diabetes case management for low-income populations. Health Serv Res 2007;42:1943-59.

34 Gilmer TP, Philis-Tsimikas A, Walker C. Outcomes of project Dulce: a culturally specific diabetes management program. Ann Pharmacother 2005;39:817-22.

35 von Elm E, Altman DG, Egger M, et al. The strengthening the reporting of observational studies in epidemiology (STROBE) statement: guidelines for reporting observational studies. J Clin Epidemiol 2008;61:344-9.

36 Carias C, Chesson HW, Grosse SD, et al. Recommendations of the second panel on cost effectiveness in health and medicine: a reference, not a rule book. Am J Prev Med 2018;54:600-2.

37 Texas Health Care Information Collection Center,. Health data researchers, and health care facilities, Texas inpatient public use data file (PUDF) 1999-2019. Available: https://www.dshs.texas.gov/thcic/ hospitals/Inpatientpudf.shtm

38 CDC National health and nutrition examination survey data 19992008, 2014. Available: http://www.cdc.gov/nchs/nhanes.htm

39 Fortmann AL, Gallo LC, Garcia MI, et al. Dulce digital: an mHealth SMS-based intervention improves glycemic control in Hispanics with type 2 diabetes. Diabetes Care 2017;40:1349-55.

40 Nichols GA, McBurnie M, Paul L, et al. The high prevalence of diabetes in a large cohort of patients drawn from safety net clinics. Prev Chronic Dis 2016;13:E78.

41 Husereau D, Drummond M, Petrou S, et al. Consolidated health economic evaluation reporting standards (cheers) statement. BMJ 2013:346:f1049.

42 Norris SL, Engelgau MM, Narayan KM. Effectiveness of selfmanagement training in type 2 diabetes: a systematic review of randomized controlled trials. Diabetes Care 2001;24:561-87. 